Revista Ingeniería y Región. 2016;16(2): 85-97

http://dx.doi.org/10.25054/22161325.1303

\title{
Análisis de posibles conflictos entre usos agrícola, rural, urbano y ambiental de los suelos
}

\section{Analysis of possible conflicts among agricultural, rural, urban and environmental uses of soil}

\author{
Yolima del Carmen Agualimpia ${ }^{1}$ y Carlos Enrique Castro ${ }^{2}$
}

\begin{abstract}
Resumen
En este artículo se expone un análisis del contraste de los resultados obtenidos en la investigación "Alternativas metodológicas para la determinación del caudal ecológico como estrategia para un desarrollo sostenible en fuentes superficiales de agua" desarrollado en el año 2008, por el grupo PROGASP de la Universidad Distrital Francisco José de Caldas y el artículo "Análisis de proyección de la demanda de agua para el uso agrícola en un sector de la sabana de Bogotá-Colombia" (Castro y Agualimpia, 2015). Mediante un análisis comparativo entre escalas se realizó la identificación de Suelos Hidro-Conductores, los cuales cumplen la función ecosistémica de permitir el flujo superficial de agua para mantener los caudales ambientales y generar un equilibrio ecológico en un sector plano de los municipios de Cota, Tabio y Tenjo. Se explora la aplicación de la norma de usos aplicada en los esquemas de ordenamiento territorial rural y urbano confrontados con dos enfoques institucionales que normativamente también deciden el uso agrícola y el ambiental.
\end{abstract}

En la actualidad se toma la decisión de cambio de uso de agrícola a urbano mediante la aplicación del Decreto 3600 de 2007 del Ministerio de Ambiente y Vivienda y Desarrollo Territorial específicamente en predios que clasifican en clases agrológicas superiores a 4 , y que cumplen con el servicio ecosistémico de regular las aguas subsuperficiales; al no ser explícito sobre cómo proceder con estas tierras, hay posibilidad de que en este territorio se configuren conflictos ambientales y sean extensivos a la totalidad de la Sabana de Bogotá.

Palabras clave: conflictos de uso; uso rural; uso agrícola; uso urbano, uso ambiental; clase agrológica; suelos hidro-conductores.

\begin{abstract}
This article presents an analysis of the contrast of the results obtained in the research entitled "Methodological alternatives for the determination of the ecological flow as a strategy for a sustainable development in surface water sources" developed in 2008 by the PROGASP group of
\end{abstract}

1 Grupo de Investigación PROGASP-Universidad Distrital Francisco José de Caldas. Doctora en ciencias técnicas. y agualimpia. Correo electrónico: yagualimpiadualiby@gmail.com

2 Grupo de Investigación PROGASP. Universidad Distrital Francisco José de Caldas. Instituto Geográfico Agustin Codazzi. Subdirección de Agrología. Magíster en geografía. Correo electrónico: cecastro77@gmail.com 
the Universidad Distrital Francisco José de Caldas and the article "Analysis of projection of water demand for agricultural use in a sector of the Sabana de Bogotá" (Castro and Agualimpia, 2015).

Through a comparative analysis between scales, Hydro-Conductive Soils, Hydro- Conductive Soils, which fulfill the ecosystem function of allowing surface water flow to maintain environmental flows and generate an ecological balance in a flat sector of the municipalities of Cota, Tabio and Tenjo, were identified. It explores the application of the norms of use applied within rural and urban terroritorial legal framework and use schemes explored according to two institutional focuses that normally decide agricultural and environmental use.

At the moment the decision is made to change from the use of agricultural soil to urban soil as stipulated in Decree 3600 of 2007. This Decree specifically refers to properties that classify in agrological classes higher than 4 . These properties comply with the ecosystem service to regulate the water's subsurface; as it is not explicit about how to proceed with these lands, there is a possibility that in this territory there will be environmental conflicts extending to the whole of the Sabana of Bogota.

Keywords: use conflicts; rural use; agricultural use; urban use; environmental use; agrological class; hydro-driver soils.

\section{Introducción}

La conservación de la biodiversidad en humedales podría incluir los suelos de drenaje imperfecto debido a que cumplen la función de conectar los flujos de agua subsuperficial y permitirles un servicio ambiental que a la vez genera funcionalidad productiva. La adecuación de suelos pobremente drenados a la agricultura intensiva a través del drenaje, en algunos casos produce impactos al ambiente al formar suelos salinos en planos de inundación del río en clima cálido seco (Cuervo, 2012) o suelos sulfato-ácidos en depresiones montañosas que acumulan materia orgánica en clima frío seco (Rincón, et al, 2008).

Cuando se ordena el uso en el territorio se debe tomar decisiones coherentes con el paradigma de la sostenibilidad de manera que permita decidir entre lo productivo y lo ambiental para evitar los errores de planificación sucedidos en el pasado.

Según IGAC (2016a,b) el uso agrícola o productivo es aquel que se desarrolla en suelos con aptitud general para cultivos; el suelo rural certificado con clases agrológicas 1,2 y 3 es reservado para actividades agrícolas mediante el Decreto 3600 de 2007 del Ministerio de Ambiente y Desarrollo Sostenible para dar seguridad alimentaria al país. La norma establece que los suelos de clases superiores a 3 podrían cambiar de agrícola a urbano, pero establecen algunas excepciones a este cambio en categorías que pueden afectar la comunidad, caso de áreas para conservación de los recursos hídricos, control de procesos erosivos o protección de zonas forestales.

Uso ambiental es aquel que se sigue para satisfacer las necesidades generales de la población, para el disfrute escénico y mantenimiento de la biodiversidad; en este sentido el suelo es valorado por la función ecosistémica de producir biomasa, ciclado de elementos, reservorio de genes, conservación del patrimonio histórico, regulación climática entre otros servicios (Ministerio de Ambiente y Desarrollo Sostenible, 2014). En los planes de ordenamiento territorial se identifican estos espacios como suelos de protección, en los cuales se involucran además las tierras con amenaza por inundación (IGAC, 2003).

En otro aspecto se encuentran los enfoques rural y urbano que en cierta manera confunden, pues desde el ordenamiento territorial un suelo rural puede ser o no agrícola y puede pasar a urbano si cumple algunos requisitos de infraestructura y de uso insostenible en agricultura. Los usos urbanos también pueden ser sustentados bajo la necesidad imperante de beneficio para todos en la etapa de concertación de los esquemas de ordenamiento territorial y a través del modelo de desarrollo se solicita el cambio de uso de rural a urbano. En última instancia los conflictos se pueden generar tanto para las comunidades que dependen de los espacios rurales, urbanos o en casos extremos ambos podrían verse afectados por amenazas ambientales. 
El uso rural es una actividad que se cumple en espacios abiertos que pueden incluir agricultura de menor escala, ganadería o usos mixtos en contraste el uso urbano se establece en los planes o esquemas de ordenamiento territorial, mediante la presencia de infraestructura vial, red primaria, servicios domiciliarios y aptitud civil para la construcción.

El cambio hacia un mundo globalizado trae conflictos territoriales que afectan directa o indirectamente las comunidades que se encuentran asentadas. Tal fue el caso analizado por Gazzano, et al, 2016, quienes hallaron contradicciones en las formas de gestión del sistema ambiental para la cual definieron dos territorios afectados por el conflicto causado por el ingreso de commodities al Uruguay, las tierras altas destinadas a la agricultura intensificada y las tierras bajas asignadas a la protección. En Bogotá, Colombia se establecen tensiones en las interfaces urbano-rural cuando se desplazan las industrias a los municipios cercanos y se decide el uso mediante el desarrollo de vías perimetrales y establecimiento de megaproyectos que conducen a decisiones no consultadas con la comunidad (Ramírez, 2009).

Un conflicto se presenta cuando el uso que se aplica al suelo, no corresponde con la vocación asignada mediante normas; en este sentido Molina, et al, 2007, encontraron que en el sector del Nula, estado Apure en Venezuela, es necesaria una gestión planificada debido a que el uso que realizan los inmigrantes colombianos no corresponde con la capacidad agrológica y en algunos casos, utilizan tierras de ecosistemas frágiles incluidas las del Parque Nacional el Tamá. En relación con la invasión de áreas protegidas Avellaneda, et al, 2015, identificaron conflictos entre normas ambientales vigentes y usos actuales en zonas de reserva, para lo cual propusieron alternativas para los habitantes de páramo.

La expansión urbana genera un conflicto en el medio ambiente debido a que se combinan los usos del suelo en la periferia y producen un desorden en las actividades suburbana, agrícola, industrial o comercial; Galfioni, et al, 2013, determinaron que esta combinación de usos impacta directamente los recursos hídricos de que dispone la comunidad de río Cuarto en Argentina.

Rubiano et al (2008) establecieron que la relación entre población y ambiente está dirigida por intereses de grupos económicos que definen el uso de las tierras a través de los instrumentos de planificación, esto oca- siona un desbalance social en los territorios. Las relaciones de poder configuran conflictos al proponer la idea del desarrollo, sin mediar las consecuencias que esto pueda traer al medio ambiente. El territorio es entonces considerado por Massiris (2009), como un espacio apropiado por grupos sociales e instituciones que ejercen dominio a partir de diversos modos de territorialidad. La ordenación de los usos del territorio se genera a partir de la apropiación de espacios como resultado de las decisiones de poder, que se institucionalizan en planes o esquemas de ordenamiento territorial.

Córdova, et al, 2016, establecieron que en San Miguel de Lules en la provincia de Tucumán en Argentina, se configura un proceso de desarrollo territorial "polarizado, diversificado y fragmentado"; las actividades que degradan los ecosistemas son toleradas por las autoridades locales debido a la ausencia de instrumentos de control del uso, corrupción local, olvido del estado y poco interés en el bienestar de la población.

La biodiversidad como estrategia ordenadora del territorio fue trabajada por Pérez, et al, 2011; encontraron que las políticas de conservación establecidas por las autoridades generan conflictos en la comunidad de San Sebastián de Palmitas y San Cristóbal en Medellín Colombia, por lo cual proponen alternativas de intervención basadas en la relación sociedad-naturaleza, de modo que sean concertadas con la población rural a través de la inclusión de variables ambientales y territoriales.

La planificación del territorio puede realizarse desde la ciencia de la ecohidrología para evitar los conflictos ambientales por el agua; permite contextualizar el uso bajo los objetivos social, económico y ambiental para asegurar la sostenibilidad de los ecosistemas mediante prácticas de concertación (González, et al, 2013).

El enfoque ambiental en Colombia se encuentra mediando entre dos usos establecidos en los planes y esquemas de ordenamiento territorial el urbano y el rural, mientras que en las áreas suburbanas se deja abierta la posibilidad de crear territorio urbano, debido a que estos espacios cuentan con algunos servicios domiciliarios (IGAC, 2003). Los enfoques aplicados al uso de las tierras por la institucionalidad colombiana presentan un divorcio que genera conflictos en las tierras; el enfoque productivo mediado en la capacidad agrológica de las tierras y el enfoque ambiental a través de normas que aplican las Corporaciones Autónomas Regionales. 
Para la ordenación ambiental del territorio colombiano se trabaja el concepto de servicio ambiental del suelo, el cual en la actualidad no es aplicado de forma transversal e integral en el ordenamiento urbano o rural, esto causa problemas de fragmentación y amenaza la sostenibilidad del territorio; los usos agrícolas cumplen una función ambiental menor (Instituto de Investigación de Recursos Biológicos Alexander von Humboldt, 2014) y en casos de sobreutilización puede impactar el caudal base de los ríos debido a que los cultivos consumen agua con mayor eficiencia que la vegetación natural (Chapagain, et al, 2003).

La calidad del suelo para agricultura está dada por la concentración y tipo de materia orgánica en la capa arable (Swift, et al, 1993 y Ferreiro, 2006). El movimiento de agua en el suelo depende de la velocidad de concentración de las aguas en sitios denominados en esta investigación Suelos Hidro-Conductores, de aquí que como hipótesis de trabajo se cree que hay una estrecha relación entre los usos agrícolas y ambientales que debe ser trabajada y normalizada para evitar posibles conflictos en el territorio. Las decisiones en muchas ocasiones se toman de manera unilateral a beneficio de uno $u$ otro enfoque, lo cual no permite cumplir con el paradigma actual de sostenibilidad.

Los conflictos se generan de planificaciones y políticas equivocadas cuando se desconoce la relación existente entre los enfoques productivo y ambiental; el uso agrícola depende del ambiental, aunque se crea que la adecuación de tierras es la solución a las necesidades de alimento, el impacto al ambiente puede ser mayor.

El objetivo es explorar el concepto transversal de los conflictos de uso en un sector de la sabana mediante la identificación de Suelos Hidro-Conductores y confrontación con los usos actuales en dichos sectores, para identificar los posibles conflictos de uso entre los usos ambiental, agrícola, urbano y rural.

Se realiza la propuesta de normalizar los Suelos HidroConductores para corregir los errores generados en la aplicación, que ha llevado a fragmentar los servicios ambientales que cumplen estos suelos y la disminución del caudal base de los ríos de la región.

El estudio general de suelos de Cundinamarca (IGAC, 2000a,b,c), se tomó como fuente de información para el esquema de ordenamiento territorial de los munici- pios de la sabana de Bogotá; las unidades principales de paisaje fueron el lomerío y la planicie fluvio lacustre, esta última con predominio de suelos derivados de cenizas volcánicas (andisoles) considerados clase 2, cuya limitante principal eran las frecuentes heladas. Las decisiones sobre el cambio de uso agrícola a urbano entre 2000 y 2007 fueron concertadas durante el esquema de ordenamiento territorial. Entre los años 2007 y 2012 los cambios de uso se realizaron bajo el Decreto 3600 de 2007 pero con criterio del estudio general de suelos.

La presión por el uso de las tierras para asentamientos urbanos llevó a la realización de estudios de suelos en mayor detalle y se escogió la escala 1:10.000 como instrumento que permitiría la planificación del uso de la tierra. A esta escala se pueden identificar suelos que tienen importancia hídrica; se trazan unidades geomorfológicas distribuidas en espacios relativamente altos y se caracterizan los suelos mediante observaciones directas en el territorio.

Los suelos agrícolas de la sabana de Bogotá abarcan una extensión de 88100 ha. (IGAC, 2000a,b,c); de acuerdo con la implementación del Decreto 3600 de 2007 del Ministerio de Ambiente, fue necesario revisar las clases agrológicas 2, 3 y 4 a escala de mayor detalle y durante 2009 y 2012 se eligieron catorce municipios (Bojacá, Cajicá, Chía, Cota, Facatativá, Funza, Gachancipá, Madrid, Mosquera, Soacha, Sopó, Tabio, Tenjo y Tocancipá) de la sabana de Bogotá, cercanos a la capital colombiana, para definir las unidades de capacidad de uso de las tierras a la escala 1:10.000. Los resultados confirmaron que 17968.23 ha tenían los mejores suelos clasificados en clase $2 ; 19626$ ha fueron incluidas en la clase agrológica 3 lo cual corresponde al $56.5 \%$ del área y debía constituirse en una zona de reserva agrícola debido a la calidad de sus suelos para este tipo de uso (IGAC, CAR y MINAMBIENTE, 2012).

Se considera que la clase agrológica 4 corresponde a la marginal y cubre 15499.12 ha $(23.3 \%)$ en estos catorce municipios; la clase agrológica 5 se extiende en una área de 5953.76 ha (9\%) y las clases 6 y 7 que no estaban identificadas en el estudio general del suelos IGAC (2000a,b,c), abarcan 4406.68 ha. Las clases agrológicas superiores a 4, al no tener aptitud para agricultura intensiva, podrían ser liberadas para otros usos en los respectivos planes y esquemas de ordenamiento territorial de los municipios estudiados. 
Las áreas identificadas con capacidad de uso superior a clase 4 que cumplen funciones ecosistémicas tiene un porcentaje aprox. de $32 \%$, sobre estas unidades puede presentarse un conflicto ambiental, ocasionado por usos agrícolas o urbanos.

\section{Materiales y métodos}

Para conocer los posibles conflictos generados por decisiones tomadas en cuanto a los usos agrícolas ambientales o urbanos, se eligió un sector plano que cubre los municipios de Cota, Tabio y Tenjo en una extensión de 15587.9 ha localizados entre las coordenadas $4^{\circ} 56$ ' 39.472 " norte, 4 $4^{\circ} 43^{\prime} 21.409^{\prime}$ 'sur, $74^{\circ} 04^{\prime}$ 46.659" este y $74^{\circ} 13$ ' 10. 998 "oeste (Figura 1).

Se realizó un análisis comparativo de la información existente en el tema de clases agrológicas producida por el Instituto Geográfico Agustín Codazzi a escala 1:100.000 (IGAC, 2000a,b,c) y escala 1:10.000 (IGAC, 2012), con el fin conocer las visiones cartográficas antes y después de la expedición del decreto 3600 de 2007.
La información detallada de suelos producida por el IGAC, CAR y MINAMBIENTE (2012) fue evaluada mediante métodos de calidad de suelos para conocer el grado de correspondencia con los espacios agrícolas establecidos en la cartografía de clases agrológicas. Se tomaron y evaluaron 83 muestras de suelo hasta $30 \mathrm{~cm}$ de profundidad. El dato hallado de carbono orgánico se llevó a información de materia orgánica mediante la aplicación del factor 1,72 el resultado georeferenciado se sometió a krigging por el método inverso de la distancia del paquete Arc Gis.

El siguiente paso fue la evaluación de los productos a las dos escalas y la delimitación de los Suelos HidroConductores que tienen como característica la afectación por encharcamientos, inundaciones, procesos de óxido reducción por fluctuación del nivel freático, a través del análisis de fases cartográficas y regímenes ácuico o integrado ácuico.

En campo se identificaron los procesos de óxido reducción en unidades puntuales representadas en la cartografía de suelos 1:10.000 (IGAC, CAR y

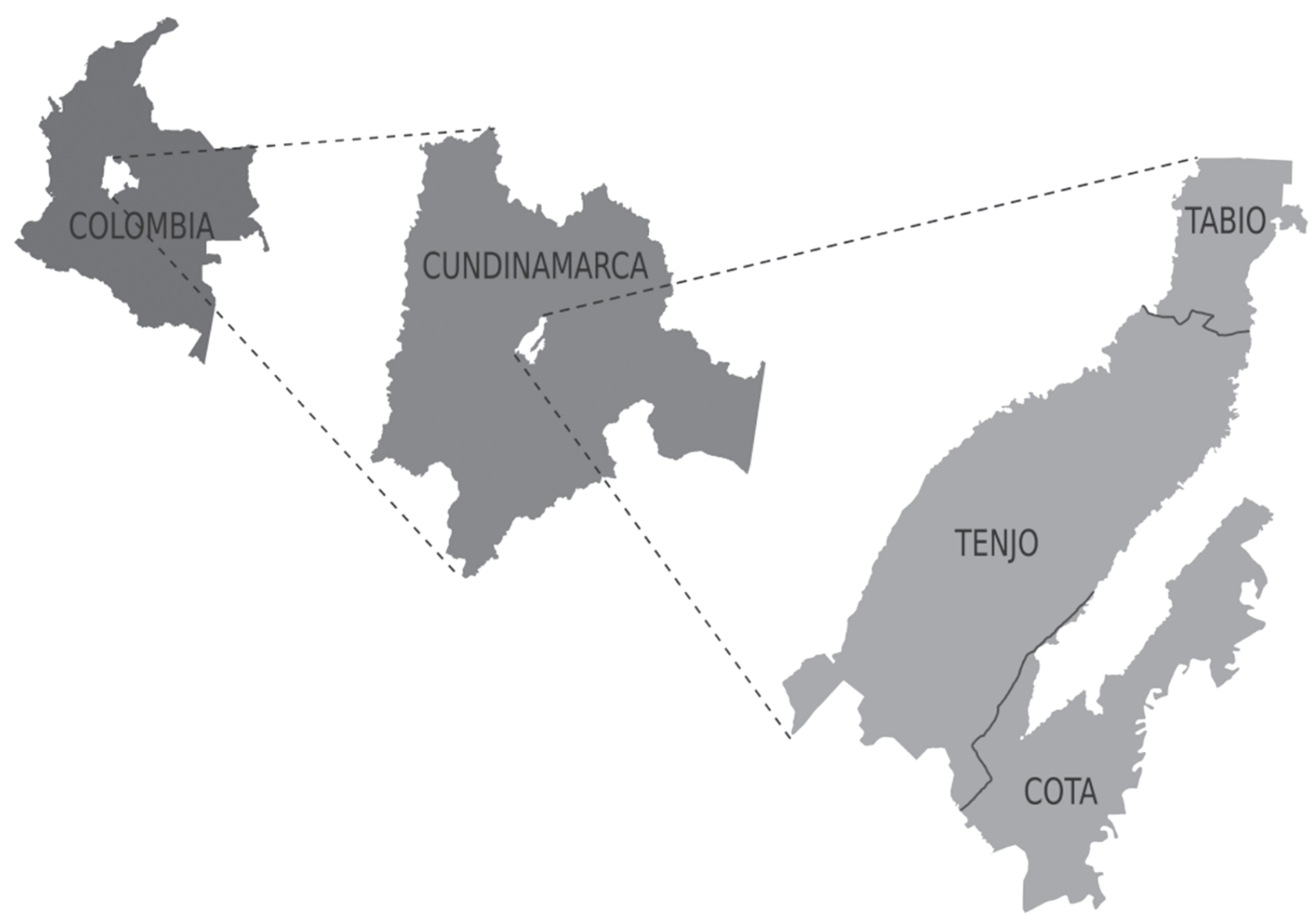

Figura 1. Localización del área de estudio en Cundinamarca-Colombia 
MINAMBIENTE, 2012) y se realizaron diez pruebas de infiltración y diez de conductividad hidráulica en los Suelos Hidro-Conductores para caracterizar el movimiento y evaluar la eficiencia del servicio ambiental que cumple.

Para identificar el posible conflicto entre el uso agrícola y ambiental se realizó un análisis comparativo del resultado de los Suelos Hidro-Conductores, el procedimiento consistió en verificar los cambios de uso que generan dichos conflictos, a través de la superposición del producto generado (Suelos Hidro-Conductores) en imágenes satelitales y corroboración con visita a campo.

El conflicto entre lo urbano y lo rural se determinó mediante la confrontación de estos resultados con los esquemas de ordenamiento territorial y la presencia de conjuntos residenciales en zonas que según los estudios detallados de suelos contenían Suelos Hidro-Conductores. Como soporte se buscaron fuentes alternas basadas en otras investigaciones incluidos los estudios antropológicos realizados en la región.

\section{Resultados}

De la comparación de clases agrológicas escala 1:100.000 con las obtenidas a escala 1:10.000 se encontró que en la zona estudiada de Cota, Tabio y Tenjo, 253,3 ha aún se constituyen en cuerpos de agua. Los procesos de ocupación generados con la presencia de población modificaron el espacio natural y a la escala detallada $(1: 10.000)$ se delimitaron 147 ha concernientes a zonas de relleno que no presentan posibilidad de uso agrícola y podían ser cambiadas a uso urbano; estas áreas de relleno se localizan principalmente en el municipio de Cota.

En las áreas planas de los municipios de Cota, Tabio y Tenjo (15587.9 ha) se logró establecer que el 74.66\% correspondía a clases agrícolas (IGAC, CAR y MINAMBIENTE, 2012).

Se realizó un análisis espacial comparativo entre las clases agrológicas y calidad de suelos para evaluar la distribución de los suelos agrícolas; con la información georeferenciada de 83 muestras de suelo se aplicó un modelo predictivo utilizando la herramienta de análisis espacial del paquete Arc gis, para establecer los sitios de mayor concentración de materia orgánica, elemento del suelo que se constituye en un referente de aptitud óptima para los cultivos (Figura 2).
Se encontró que en el municipio de Tenjo hay una alta correlación de la calidad del suelo representado en la capacidad de uso agrícola; es decir, mayor cantidad de materia orgánica (13-15\%) en suelos de la clase agrológica 2, lo cual se interpreta que estas áreas rurales deben conservarse para agricultura debido a su alta calidad.

La alta capacidad de retención hídrica asociada a los contenidos de materia orgánica, fueron contrastados con los resultados presentados por IGAC, CAR y MINAMBIENTE, (2012); se encontró que los suelos agrícolas pueden saturarse con agua incluso por encima de su peso, demostrando así su alto poder de retención de humedad, al igual que la función ecológica de regular altos volúmenes de agua. Al analizar la saturación de agua de las muestras de suelos, se encontró que presentaban valores entre 81.82 y $142.47 \%$ y la humedad aprovechable variaba de 9.85 a $32.74 \%$ con un valor promedio de $23.16 \%$; es decir los suelos tienen agua retenida a presión tal que las plantas son capaces de extraerla para sus funciones fisiológicas; este valor promedio es considerado alto (IGAC, 1990).

Determinada la representatividad de los suelos agrícolas se procedió a analizar las bases de datos del suelo del estudio IGAC, CAR Y MINAMBIENTE (2012), para identificar aquellos suelos que cumplen una función ecosistémica que beneficia tanto los procesos productivos como los ambientales. El análisis del paisaje permitió deducir que en las partes proximal, medial y distal del glacis de acumulación el drenaje natural varía entre moderado a pobre desde la parte alta colindando con la ladera del lomerío a la base en límite con las terrazas lacustres intermedia e inferior (Tabio y Tenjo). A medida que se baja en el relieve se llega a la planicie de inundación en límites con el río Bogotá en el municipio de Cota, cuyos servicios ecosistémicos son más claros y representan áreas inundables y encharcables con régimen de humedad ácuico.

El municipio de Tabio se caracteriza por poseer áreas con Suelos Hidro-Conductores en el sector relativamente alto y representativo de la sabana de Bogotá, lo cual indica su importancia y provisión de agua freática para los procesos productivos que suceden en los paisajes más bajos.

El proceso cartográfico con el cual se delimitaron los Suelos Hidro-Conductores representa la función ambiental. Mediante salida a campo se verificaron los procesos de óxido reducción para ello se utilizó el barreno holandés, se estudió el drenaje con la tabla de 

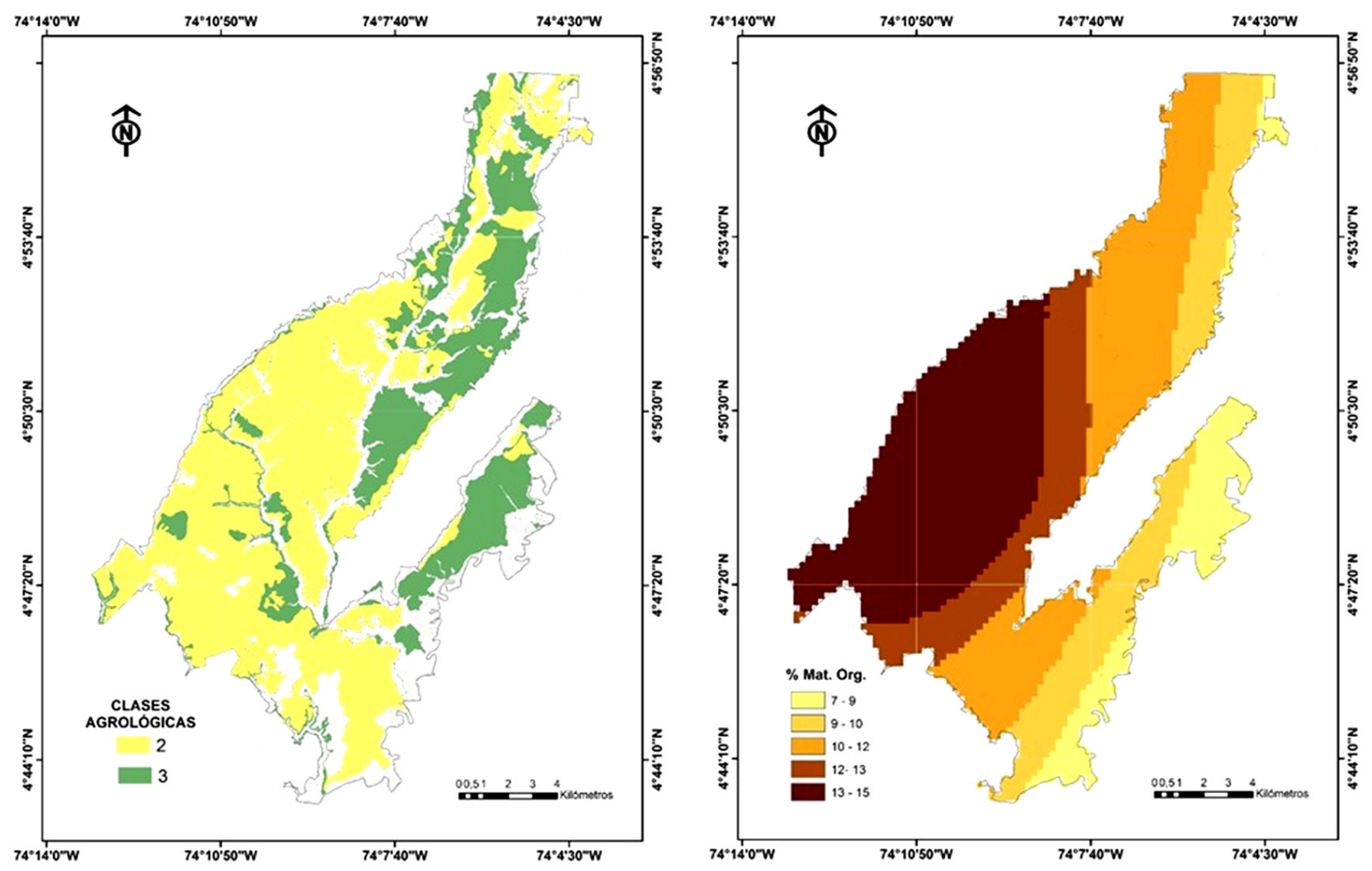

Figura 2. Comparación de clases agrológicas con el porcentaje de materia orgánica.

(IGAC, CAR Y MINAMBIENTE, 2012).

colores Munsell y se observaron niveles freáticos. Se escogieron diez puntos representativos de los paisajes y se realizaron pruebas de infiltración y conductividad cuyos resultados se presentan en la tabla 1.
Los suelos que cumplen funciones ambientales tienen características especiales tales como humedad excesiva, niveles freáticos altos, texturas arcillosas, inundación o encharcamiento frecuentes. Se realizó un

Tabla 1. Movimiento del agua en los paisajes que contienen Suelos Hidro-Conductores.

\begin{tabular}{c|c|c|c|c|c|c}
\hline \multirow{2}{*}{$\begin{array}{c}\text { Tipo de Suelos } \\
\text { Hidro-Conductores } \\
\text { (SHC) }\end{array}$} & Forma de terreno & Drenaje natural & Conductividad hidráulica, cm/h & \multicolumn{2}{c}{ Infiltración, cm/h } \\
\cline { 5 - 7 } & & & K & Calificación & $\begin{array}{c}\text { Infiltración } \\
\text { básica }\end{array}$ & Calificación \\
\hline SHC-1 & Proximal - Glacis & Moderado & 3.00 & Moderada & 4.80 & Moderada \\
\hline SHC-2 & $\begin{array}{c}\text { Bajo - Terraza } \\
\text { media }\end{array}$ & Pobre & 0.30 & Lenta & 0.20 & Lenta \\
\hline SHC-3 & $\begin{array}{c}\text { Plano alto - } \\
\text { Terraza media }\end{array}$ & Moderado & 0.64 & Moderada Lenta & 0.52 & Moderada Lenta \\
\hline SHC-4 & $\begin{array}{c}\text { Plano medio- } \\
\text { Terraza media }\end{array}$ & Moderado & 2.11 & Moderada & 2.05 & Moderada \\
\hline SHC-5 & $\begin{array}{c}\text { Plano bajo - } \\
\text { Terraza media }\end{array}$ & Moderado & 0.70 & Moderada Lenta & 0.52 & Moderada Lenta \\
\hline SHC-6 & $\begin{array}{c}\text { Bajo - Terraza } \\
\text { media }\end{array}$ & Pobre & 0.03 & Muy Lenta & 0.01 & Muy Lenta \\
\hline SHC-7 & $\begin{array}{c}\text { Bajo - Terraza } \\
\text { inferior }\end{array}$ & Pobre & 1.40 & Moderada Lenta & 1.62 & Moderada Lenta \\
\hline SHC-8 & $\begin{array}{c}\text { Bajo - Terraza } \\
\text { media }\end{array}$ & muy pobre & 0.75 & Moderada Lenta & 0.72 & Moderada Lenta \\
\hline SHC-9 & cubeta de desborde & Pobre & 0.42 & Lenta & 0.42 & Lenta \\
\hline SHC-10 & $\begin{array}{c}\text { cubeta de } \\
\text { decantación }\end{array}$ & muy pobre & 0.51 & Moderadamente Lenta & 0.14 & Lenta \\
\hline
\end{tabular}


análisis de los suelos que tienen régimen de humedad ácuico, incluidos los integrados ácuicos y se encontró que 3139.4 ha (20.14\%) se constituían en suelos que representan la conectividad de procesos hidrológicos asociados al almacenaje y tránsito de aguas superficiales en el paisaje de sabana (figura 1).

El análisis integral de los datos de infiltración y conductividad hidráulica (Tabla 1) arrojó valores promedio de cómo se mueve el agua en depresiones del paisaje constituidas por las formas de terreno de partes proximal, medial y distal del glacis de acumulación, bajo y plano de las terrazas lacustres, vallecitos y cubetas de la planicie de inundación del río Bogotá.

Los aportes de lluvia se infiltran en estos paisajes a una velocidad promedio de $1.10 \mathrm{~cm} / \mathrm{h}$, que se consideran moderadamente lenta, lo cual conduce a un flujo superficial efectivo y se mueve a través de él a una velocidad promedio de $0.99 \mathrm{~cm} / \mathrm{h}$ que se considera también un movimiento moderadamente lento según IGAC (1990).

\section{Posibles conflictos}

Para identificar los posibles conflictos en suelos agrícolas, se aplicó el enfoque productivo para lo cual se preparó la cartografía de clases agrológicas (IGAC, CAR Y MINAMBIENTE, 2012), validada mediante índices de calidad del suelo determinada en la evaluación y distribución de porcentajes de materia orgánica; se encontró que en el sitio cinco en el municipio de Cota (Figura 3), los suelos agrícolas clases 2 fueron ocupados con infraestructura urbana y bodegas a lo largo de la salida de Bogotá. Esta decisión se tomó durante la actualización del esquema de ordenamiento en el período 2007 a 2011, por tanto la información cartográfica escala 1:100.000 desde ese entonces caracterizaba el sector con suelos óptimos para agricultura.

Los posibles conflictos ambientales se identifican mediante un enfoque diferente al productivo y a través del cual se da importancia a los servicios ambientales que prestan los Suelos Hidro-Conductores pues transfieren agua y nutrientes a través de los paisajes.

El sector alto de la zona de estudio en el municipio de Tabio, se encuentra con amenaza de conflicto entre ambiental y urbano, pues en el sitio uno (Figura 3) se presenta la posibilidad de fraccionamiento del paisa- je debido a que la zona se encuentra rodeada de vías, lo que puede causar la adecuación o relleno para establecimiento de viviendas. El paisaje se localiza en los bajos y plano de la terraza media y constituye el sitio de recarga de aguas subsuperficiales que fluyen hacia el río Chicú. La amenaza de posible conflicto se encuentra en el sector la Esperanza en la finca Santa Helena y la esperanza Lourdes en Tabio Cundinamarca.

El aprovechamiento de agua desde el nacimiento del río Chicú, causa un posible conflicto agrícola-ambiental en el sitio dos (Figura 3), que corresponde al municipio de Tenjo.

El establecimiento de invernaderos cercanos al río Chicú no permite controlar la cantidad de agua que se extrae y esto le convierte en una amenaza para el caudal ambiental. El conflicto rural en este caso es al ambiente pues la utilización excesiva de agua y la contaminación con fertilizantes que causan nitrificación en las aguas, disminución de la población de macroorganismos acuáticos y desaparición de las especies ícticas.

El posible conflicto de estas características se identificó en áreas del municipio de Tenjo en el lugar que se establecieron los cultivos de flores cerca del casco urbano de Tenjo, en la vereda el Chacal y en el sitio Santa Catalina, vista panorámica al sector el camping.

Un posible conflicto derivado del enfoque ambiental, se identifica en el sitio tres del municipio de Cota (Figura 3) y se refiere a una decisión tomada antes de la expedición del decreto 3600 de 2007, durante el primer esquema de ordenamiento territorial y con base en la información de clases agrológicas escala 1:100.000; aunque la cartografía no aplicaba para cambiar el uso, no deja de ser un inconveniente producto del crecimiento urbano. La ocupación del plano de inundación del río Bogotá y el control de desbordamientos con diques construidos ocasiona la disminución en la eficiencia en que estas áreas regulan los caudales naturales.

Al observar los beneficios ambientales como un todo integrado y articulado mediante interconexiones hídricas de redes y humedales, se pueden conocer los servicios ecosistémicos que cumplen los flujos subsuperficiales a la flora natural o intervenida. El enfoque ambiental es totalizante e involucra las prácticas de protección a los remanentes húmedos que aún subsisten en la sabana de Bogotá. 


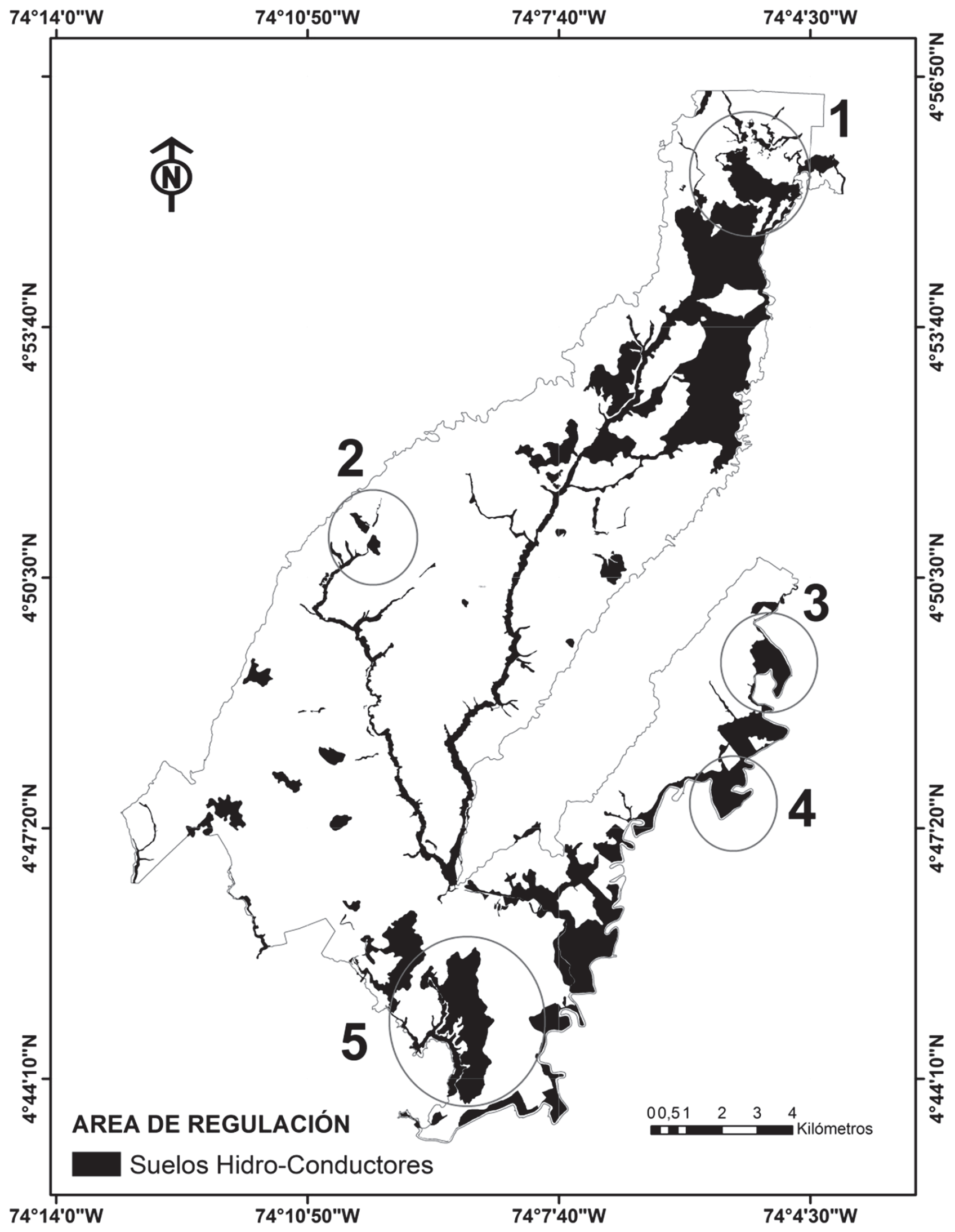

Figura 3. Distribución de los Suelos Hidro-Conductores y localización de sitios con posibles conflictos (IGAC,CAR Y MINAMBIENTE, 2012). 
El posible conflicto de uso en el sitio cuatro (de la figura 3 ) se debe a la ocupación de tierras de uso ambiental, incluso aquellas tierras que guardan la memoria cultural y estudio del hombre del altiplano; este lugar constituye sitios cerrados para disfrute de una comunidad exclusiva.

El sitio cuatro también se encuentra afectado ambientalmente por el uso agrícola dado que hay presencia de invernaderos muy cerca de la margen izquierda del río, lo cual no permite el control del aprovechamiento de agua y puede ser excesivo para estos espacios de la sabana de Bogotá tan necesitados de agua.

Un posible conflicto ambiental-urbano se genera cuando se construyen vías en tierras que continen suelos mal drenados proximos a un humedal. El proceso de ocupacion en zonas suburbanas terminan por drenar estas tierras (sitio cinco de la Figura 3).

En el bajo de la terraza inferior en límites con el río Bogotá en el municipio de Cota, se presenta la ocupación de suelos mal drenados con bodegas. Un brazo del río fue clausurado y canalizado para controlar la inundación. La entrada a Bogotá por la calle ochenta generó la ocupación de terrenos cerca del lago en el parque de la florida, el cual con el humedal Gualí hacían parte del complejo de sistemas hídricos que se surtían de agua de las crecientes del río Bogotá.

El posible conflicto ambiental-urbano se configura con la construcción de terminales terrestres e instalación de centros empresariales en sitios donde se daban procesos ambientales, esto con el tiempo puede afectar la biodiversidad del lago de la florida.

Cuando se realiza un análisis general ambiental de los Suelos Hidro-Conductores a través del área de estudio y se identifica su estructura y función se puede conocer los servicios ecosistémicos que cumplen este tipo de suelos y tener una visión crítica de los cambios de uso que alteran el funcionamiento de los ecosistemas naturales y productivos, los cuales en última instancia afectan las comunidades humanas.

\section{Discusión}

Hay un vacío en la aplicación de la normatividad vigente, en cuanto a la protección de los Suelos HidroConductores, debido a que no se menciona de manera explícita en el decreto 3600 de 2007. La identificación de las clases agrológicas 1,2 y 3 permite garantizar la producción agrícola, seguridad alimentaria; de igual importancia la conservación de los Suelos Hidro-Conductores, permite disminuir las amenazas naturales por inundación, debido a que estos sectores cumplen servicios ecosistémicos basados en el tránsito, almacenaje y regulación del caudal.

El enfoque de la sostenibilidad no ha sido implementado en las decisiones del uso que se aplican en la Sabana de Bogotá. La visión productiva ha permitido conservar los suelos agrícolas que estaban siendo invadidos por usos urbanos; no obstante, la planeación regional puede afectar los Suelos HidroConductores que se consideran no aptos para la agricultura por ser mal drenados y de clase agrológica entre 4 y 7 ; estos suelos cumplen funciones ecológicas de almacenamiento, regulación y transporte de aguas subsuperficiales.

La escala detallada permite conocer las limitantes del suelo para el uso agrícola en detrimento del conocimiento de la función ecológica que es de escala general. La escala grande causa el olvido de los suelos que cumplen servicios ecosistémicos, debido a que su enfoque está orientado a la producción.

Es importante que lo ambiental sea observado como un todo, articulado con interconexiones hídricas entre humedales, dado que los flujos subsuperficiales benefician la flora natural e intervenida. Las soluciones deben abarcar un enfoque totalizante en donde se involucren medidas de protección a los remanentes húmedos de la Sabana de Bogotá.

Los estudios recientes han permitido el cambio de uso de suelos que eran considerados agrícolas, bajo la premisa de que la clase agrológica determinada por el IGAC era superior a 4; esto causa la fragmentación del paisaje y recorte de los flujos superficiales de agua, lo cual genera el drenaje de suelos localizados aguas abajo. Las medidas de protección de estos suelos se suscriben a grandes cuerpos de agua identificados en la convención RAMSAR, sin hacer mención de los Suelos Hidro-Conductores, cuya función es importante en el paradigma de la sostenibilidad ambiental.

Las medidas aplicadas para la protección de la biodiversidad están planteadas generalmente en la restauración de coberturas naturales, en la reforestación, en el manejo de escurrimiento de aguas y en el control de erosión. En esta investigación se plantea la protec- 
ción de los Suelos Hidro-Conductores para garantizar la sostenibilidad.

Los posibles conflictos entre los enfoques productivo y ambiental tienen el mismo soporte técnico que los enfoques de uso agrícola y urbano que se definen en los esquemas de ordenamiento territorial y para aplicar el paradigma del desarrollo sostenible deberían ser determinantes.

\section{Conclusiones}

A escala general los únicos Suelos Hidro-Conductores delimitados se encuentran en la planicie de inundación en 623 ha $(19.8 \%$ del área de estudio) y corresponden al sector más bajo y cercano al río Bogotá. A escala detallada además de las cubetas identificadas en el estudio general de suelos, se delimitaron otros Suelos Hidro-Conductores, que cumplen funciones de regulación, específicamente en los paisajes de la planicie fluvio-lacustre; 1288.7 ha (41\% del área de estudio) se encuentran en los límites entre terrazas altas, medias y bajas; 759.9 ha (24.2\% del área de estudio) están dispersas en las terrazas en la forma de terreno bajos; 372.4 ha en la vega del río Chicú y solo 96.61 ha en la parte distal del glacis de acumulación. La escala detallada permite identificar los suelos HidroConductores con mayor eficiencia que la escala general.

A partir de la comparación realizada entre el análisis morfológico y los muestreos de campo en 83 sitios escogidos estratégicamente, se encontró correlación directa entre las clases agrológicas 2 y los contenidos de materia orgánica en el suelo, lo cual garantiza una calidad adecuada para la escala, ya que el uso agrícola corresponde con suelos de abundante materia orgánica. De igual forma, existe una alta retención hídrica asociada a esta condición, lo cual demuestra una potencialidad óptima en la regulación de volúmenes de agua en los suelos agrícolas localizados en los terrenos relativamente altos.

En los municipios Cota, Tabio y Tenjo se identificaron 3139.4 ha de Suelos Hidro-Conductores equivalentes al 20.14\% del área de estudio; los suelos se caracterizan por la presencia de regímenes de humedad ácuicos o integrados ácuicos, por inundaciones o encharcamientos frecuentes y niveles freáticos altos. La delimitación de estos suelos se constituyen en el referente de servicio ambiental que representan estos suelos en la región.
Los posibles conflictos por uso agropecuario están en función de la escala a la cual es representada la capacidad de uso; en el caso de la escala general no se identifican y delimitan los Suelos Hidro-Conductores y esto tiene como consecuencia un manejo inapropiado y obstrucción de la función ecosistémica de estos suelos.

En el municipio de Cota se identificaron cuatro posibles conflictos, en el sector bajo hay un conflicto Urbano-rural por la invasión de suelos clase agrológica 2, cuyo uso cambió a urbano con la construcción de bodegas a lado y lado de la vía de entrada a Bogotá; muy cerca de allí en la vía a la florida hay un conflicto urbano-ambiental al construir bodegas en zonas mal drenadas que fueron modificadas para evitar las inundaciones y esto afectó el lago de la florida que recibe pocas aguas de crecientes del río Bogotá. El tercer posible conflicto es Urbano-Ambiental-cultural y se encuentra en el plano de inundación del río Bogotá con la dedicación de áreas para una urbanización exclusiva que posiblemente tiene artefactos de cerámica y obras construidas por la cultura chibcha y por último un conjunto residencial cerrado en el plano de inundación del río Bogotá con un conflicto ambiental-urbano.

En el municipio de Tabio se configura un posible conflicto urbano- ambiental pues está la oportunidad de que en un sector del nacimiento del río Chicú se convierta su uso ambiental a urbano debido a la presencia de vías y establecimiento de servicios básicos.

El municipio de Tenjo tiene un posible conflicto por agricultura-ambiente debido al aprovechamiento incontrolado de aguas del río Chicú.

\section{Referencias bibliográficas}

Avellaneda, L., Torres, E., León, T., 2015. Alternativas ante el Conflicto entre Autoridades Ambientales y Habitantes de Áreas Protegidas en Páramos Colombianos. En: Mundo Agrario. Abril, 16(31).

Castro, C., Agualimpia, Y., 2015. Análisis de Proyección de la Demanda de Agua para el Uso Agrícola de un Sector de la Sabana de Bogotá. Tecnogestión, una mirada al ambiente. 12:13-25.

Chapagain, A.K., Hoekstra, A.Y., 2003. Virtual Water Trade: A Quantification of Virtual Water Flows between Nations in Relation to International Trade in Livestock and Livestock Products. 
Córdova, H., Czerny, M., Novoa, Z., 2016. Ordenamiento Territorial y Desarrollo Rural. Sociedad Geográfica de Lima. Lima, Perú, p. 155.

Cuervo, R., 2012. Hacia un Sistema Complementario de Producción más Limpia en Suelos Degradados por Salinidad. En Revista del Doctorado Interinstitucional en Ciencias Ambientales. Ambiente y Sostenibilidad (2): Valle del Cauca, pp. 59-68.

Decreto 3600 de 2007. Ministerio de Ambiente, Vivienda y Desarrollo Territorial. Reglamentan disposiciones de las leyes 99 de 1993 y 388 de 1997 relativas a las determinantes de ordenamiento del suelo rural y al desarrollo de actuaciones urbanísticas de parcelación y edificación en este tipo de suelo y se adoptan otras disposiciones.

Ferreiro, J., 2006. Propiedades Bioquímicas de Suelos de Prado de Galicia. Universidad de Santiago de Compostela. Facultad de Farmacia. Departamento de Edafología y Química Agrícola, p. 515.

Galfioni, M., Degioanni, A., Maldonado, G., Campanella, O., 2013. Conflictos Socioambientales: Identificación y Representación Espacial. Estudio de caso en la ciudad de Río Cuarto (Argentina). En: Estudios Geográficos. Julio-diciembre, LXXIV(275):469-493.

Gazzano, I., Achkar, M., 2016. Conflictos de las Transformaciones Territoriales: ganaderos frente a la intensificación agraria en esteros de Farrapos Uruguay. En Revista Iberoamericana de Economía Ecológica, 26:109-121.

González, N., Hernández, M., Romero, C., 2013. La Planificación Hídrica en el Contexto de la Ecohidrología. Instituto de Cultura Jurídica y Maestría en Sociología Jurídica. FCJ y S. UNLP. En: Derecho y Ciencias Sociales. Octubre 2013. N ${ }^{\circ} 9$ (La problemática del agua en el mundo actual), pp. 15-23.

Instituto de Investigación de Recursos Biológicos Alexander von Humboldt, 2014. Principios y Criterios para la Delimitación de Humedales Continentales una Herramienta para Fortalecer la Resiliencia y la Adaptación al Cambio Climático en Colombia. Bogotá, p. 86.

IGAC, 1990. Propiedades Físicas de los Suelos. Compiladores Hugo Montenegro y Dimas Malagón. Subdirección de Agrología. Bogotá, p. 811.
IGAC, 2000a. Estudio General de Suelos y Zonificación de Tierras de Cundinamarca, Escala 1:100.000. Subdirección de Agrología. Bogotá, D.C. Tomo 1, p. 130.

IGAC, 2000b. Estudio General de Suelos y Zonificación de Tierras de Cundinamarca, Escala 1:100.000. Subdirección de Agrología. Bogotá, D.C. Tomo 2, p. 450.

IGAC, 2000c. Estudio General de Suelos y Zonificación de Tierras de Cundinamarca, Escala 1:100.000. Subdirección de Agrología. Bogotá, D.C. Tomo 3, p. 615 .

IGAC, 2003. Gestión del Suelo Urbano. En el Marco del Ordenamiento Territorial. Aproximación metodológica. Bogotá. D.C. p. 91.

IGAC, 2012. Estudio de los Conflictos de Uso del Territorio Colombiano Escala 1:100.000. Subdirección de Agrología. Imprenta Nacional. Bogotá.

IGAC, 2016a. Suelos y Tierras de Colombia. Subdirección de Agrología. Bogotá, D.C Tomo 1, p. 545.

IGAC, 2016b. Suelos y Tierras de Colombia. Subdirección de Agrología. Bogotá, D.C Tomo 2, p. 854.

IGAC, CAR y MINAMBIENTE, 2012. Levantamiento Detallado de Suelos en Áreas Planas de 14 Municipios de la Sabana de Bogotá. Escala 1:10000 Subdirección de Agrología. Bogotá, p. 492.

Ministerio de Ambiente y Desarrollo Sostenible, 2014. Política para la Gestión Integral Ambiental del Suelo. Bogotá, D.C. p. 138.

Pérez, H., Zárate, C., Turbay, S., 2011. Conflictos Ambientales: la biodiversidad como estrategia ordenadora del territorio. En: Opinión Jurídica, edición especial, Julio-Diciembre de 2011 / 166 p. Medellín, Colombia, pp. 89-104.

Rincón, A., Castro, H., Gómez, M., 2008. Caracterización física de los suelos sulfatados ácidos del Distrito de Riego del Alto Chicamocha (Boyacá) y su aplicación al manejo. En Agronomía Colombiana, 26(1):134-145.

Massiris, A., 2009. Geografía y territorio. Procesos territoriales y socioespaciales. Universidad Pedagógica y Tecnológica de Colombia. Tunja, Boyacá. p. 266. 
Molina, G., Rivas, J., 2007. Conflictos de usos normativos y agroecológicos en un área fronteriza: sector El Nula, estado Apure-Venezuela. En Revista Geográfica Venezolana, 48(1):101-114.

Ramírez, A., 2009. Análisis de los conflictos ambientales en interfases urbano-rurales Generalidades desde dos territorios de Bogotá. En Revista nodo. EneroJunio. Año 3, 6(3):71-96.
Rubiano, N., González, A., Toledo, A., Zamudio, L., Cano, C., Córdoba, C., Parra, E., 2008. Población y Ordenamiento Territorial. Universidad Externado de Colombia. Bogotá, D.C. p. 187.

Swift, M., Woomer, P., 1993. Organic matter and the sustainability of agricultural systems: Definition and measurement. In: Woomer, P.; Swift, M. (eds) the biological managemet of tropical soil fertility. Wiley, Chichester, pp. 1-12. 
